Marivaux (1752): Le Spectateur françois. Paris: Prault jeune.

Van Effen, Justus (1742): La Bagatelle ou Discours ironiques, ou l'on prête des Sophismes ingénieux au Vice \& à l'Extravagance, pour en faire mieux sentir le ridicule. Amsterdam: Herman Uytwerf.

Van Effen, Justus (1742): Le Misantrope. La Haye: Jean Neaulme/Amsterdam: Herman Uytwerf.

Van Effen, Justus (1725-1726): Le Nouveau Spectateur françois ou discours dans lesquels on voit un Portrait naïf des Mours de ce Siècle. La Haye: Jean Neaulme.

\title{
7.2 SEKUNDÄRLITERATUR
}

Abels, Heinz (2001): Interaktion, Identität, Präsentation. Kleine Einführung in interpretative Theorien der Soziologie. 2. und überarbeitete Auflage. Wiesbaden: VS Verlag.

Abels, Heinz (2009): Wirklichkeit. Über Wissen und andere Definitionen der Wirklichkeit, über uns und Andere, Fremde und Vorurteile. Wiesbaden: VS Verlag.

Ahmed, Sara (2010): „Happy Objects.“ In: Melissa Gregg/Gregory J. Seigworth (eds.): The Affect Theory Reader. Durham, NC [u.a.]: Duke University Press, 29-51.

Ammon, Sabine (2005): „Wissen verstehen. Nelson Goodmans symboltheoretische Revision des Wissensbegriffs.“ In: Andreas Arndt/Karol Bal/Henning Ottmann (eds.): Glauben und Wissen. Berlin: Akademie Verlag, 314-319.

Anderson, Benedict (2005 [1996]): Die Erfindung der Nation. Frankfurt a. M. [u.a.]: Campus.

Anderson, Paul B. (1931): „The History and Authorship of Mrs. Crackenthorpe's Female Tatler.“ In: Modern Philology 3/28, 354-360.

Aragon, Sandrine (2004): „Des révolutions dans les représentations de lectrices.“ In: Dix-huitième siècle 1/36, 237-248.

Asholt, Wolfgang/Ette, Ottmar (eds.) (2010): Literaturwissenschaft als Lebenswissenschaft. Programm - Projekte - Perspektiven. Tübingen: Narr.

Assmann, Jan (2005 [1992]): Das kulturelle Gedächtnis. Schrift, Erinnerung und politische Identität in frühen Hochkulturen. München: C.H. Beck.

Bacon, Roger (2015): Kompendium für das Studium der Philosophie. Hamburg: Meiner.

Badinter, Elisabeth (1992): XY. De l'identité masculine. Paris: Odile Jacob.

Bakanitsch, Hannah (2018): Le Spectateur français avant la Révolution (1796) von Jacques-Vincent Delacroix als Spiegel seiner Zeit. Universität Graz, Diplomarbeit.

Barnette, Linda-Jane C. (1995): „Images of Women in El Censor.“ In: Dieciocho 1/18, 77-89.

Beard, Mary (2018): Frauen \& Macht. Ein Manifest. übers. von Ursula Blank-Sangmeister, Ursula und Janet Schüffel. Frankfurt a. M.: Fischer.

Beauvoir, Simone de (1976 [1949]): Le deuxième sexe. Les faits et les mythes. Préface de Benoîte Groult. Paris: France Loisirs.

Becker, Susanne (2007): Literatur- und Kulturwissenschaften. Ihre Methoden und Theorien. Reinbek bei Hamburg: Rowohlt.

Beckers, Tilo (2016): „Werte.“ In: Johannes Kopp/Anja Steinbach (eds.): Grundbegriffe der Soziologie. Wiesbaden: VS Verlag, 373-377. 
Benjamin, Walter (1972): „Literaturgeschichte und Literaturwissenschaft.“ In: Hella Tiedemann-Bartels (ed.): Walter Benjamin. Gesammelte Schriften. Band 3. Frankfurt a. M.: Suhrkamp, 283-290.

Berger, John (2008 [1972]): Ways of Seeing. London: Penguin Books.

Berger, Peter L./Luckmann, Thomas (1991 [1966]): The Social Construction of Reality. A Treatise in the Sociology of Knowledge. London: Penguin Books.

Berlant, Lauren/Warner, Michael (1998): „Sex in Public.“ In: Critical Inquiry 2/24, 547-566.

Bernos, Marcel (1997): „Amour.“ In: Michel Delon (ed.): Dictionnaire européen des Lumières. Paris: Presses universitaires de France, 68-71.

Black, Scott (2008): „The Spectator in the History of the Novel.“ In: Media History 3/14, 337-351.

Blanchard, Rae (1929): „Richard Steele and the Status of Women.“ In: Studies in Philology 3/26, 325-335.

Bock, Gisela/Zimmermann, Margarete (1997): „Die Querelle des Femmes in Europa. Eine begriffs- und forschungsgeschichtliche Einführung.“ In: Gisela Bock/Margarete Zimmermann (eds.): Jahrbuch für Frauenforschung. Band 2. Die europäische Querelle des Femmes. Geschlechterdebatten seit dem 15. Jahrhundert. Stuttgart: Metzler, 9-38.

Böhme, Hartmut (1997): „Elemente - Feuer Wasser Erde Luft.“ In: Christoph Wulf (ed.): Vom Menschen. Handbuch Historische Anthropologie. Weinheim [u.a.]: Beltz, 17-46.

Bolufer Peruga, Mónica (1993): „Ecos de la ‘querelle des femmes’ en la España del siglo XVIII.“ In: Carlos Álvarez Santaló/Carmen María Cremades Griñán (eds.): Mentalidad e ideología en el Antiguo Régimen. Murcia: Universidad de Murcia, 185-194.

Bolufer Peruga, Mónica (1995): „Espectadores y lectoras: representaciones e influencia del público femenino en la prensa del siglo XVIII.“'In: Cuadernos de Estudios del Siglo XVIII 5, 23-57.

Bolufer Peruga, Mónica (1998a): Mujeres e Ilustración. La construcción de la feminidad en la Ilustración española. València: Inst. Alfons el Magnànim.

Bolufer Peruga, Mónica (1998b): „Culture and Gender in Spain: The Ambiguities of Enlightenment." In: History Workshop Journal 46, 261-267.

Bolufer Peruga, Mónica (2016): „Revisiter la Défense des femmes (1726) de Benito Jerónimo Feijoo.“ In: Clio. Femmes, Genre, Histoire 43, 223-249.

Bolufer, Mónica (2014): „Civilizar las costumbres. El papel de la prensa periódica dieciochesca.“ In: Bulletin of Spanish Studies 9-10/91, 97-113.

Bolufer, Mónica/Cabré, Montserrat (2015): „La Querelle des femmes en Espagne: bilan sur l'histoire d'un débat (1400-1800). “In: Armel Dubois-Nayt/Marie-Élisabeth Henneau/Rotraud von Kulessa (eds.): Discours sur l'égalitélinégalité des sexes en Europe, de 1400 aux lendemains de la Révolution // Revisiter la „,querelle des femmes “. Discours sur l'égalitélinégalité des sexes en Europe, de 1400 aux lendemains de la Révolution. Saint-Étienne: Université de Saint-Étienne, 31-67.

Bond, Donald F. (ed.) (1965): The Spectator. Volumes I-V. Oxford: Oxford University Press.

Bond, Richmond P. (1971): The Tatler. The Making of a Literary Journal. Cambridge, MA, London: Harvard University Press; Oxford University Press. 
Bony, Alain (1982): „L'Élaboration de l'auteur supposé dans l'essai périodique: Swift, Defoe, Steele et Addison. “In: Pierre Rétat (ed.): Le journalisme d'Ancien Régime. Question et propositions/ Table ronde CNRS, 12-13 juin 1981. Lyon: Presses Universitaires de Lyon, 333-349.

Bony, Alain (1999): Joseph Addison, Richard Steele. The Spectator et l'essai périodique. Paris: Didier.

Bony, Alain (2003): „Portrait du Spectator en 'Socrate moderne'“ In: Annie Cointre/Alain Lautel/Annie Rivara (eds.): La traduction romanesque au XVIIIe siècle. Arras: Artois Presses Univ, 141-164.

Boons, Hélène (2020): „Le masque brisé: heurs et malheurs de la fiction dans les 'Spectateurs' de J.-V. Delacroix de part et d'autre de la Révolution française.“ In: Klaus-Dieter Ertler et al. (eds.): Storytelling in the Spectators/Storytelling dans les spectateurs. Frankfurt a. M.: Peter Lang, 163-177.

Bösch, Frank (2011): Mediengeschichte. Vom asiatischen Buchdruck zum Fernsehen. Frankfurt a. M. [u.a.]: Campus.

Bots, Hans/Waquet, Françoise (1997): La République des Lettres. Paris: Belin.

Boulard Jouslin, Claire (2017): „'Un ordre lumineux’ ou les spécificités des essais du Spectator (1711-1714).“ In: Guilhem Farrugia (ed.): Promenade et flânerie. Vers une poétique de l'essai entre les XVIIIe et XIXe siècles. Rennes: Presses universitaires de Rennes, 81-97.

Boulard Jouslin, Claire (s. a.): „Dealing with the 'Fair Sex': Women and the Periodical Press in the Nichols Collection." In: $17^{\text {th }}$ and $18^{\text {th }}$ Century Nichols Newspapers Collection, online: https://www.gale.com/binaries/content/assets/gale-us-en/primary-sources/newsvault/gps_17th_18th_nichols_collection_-essay_jouslin.pdf, 27/03/18.

Boulard, Claire (2000): Presse et socialisation féminine en Angleterre de 1690 à 1750, conversations à l'heure du thé. Étude du Gentleman's Journal, du Spectator et du Female Spectator. Paris: L'Harmattan.

Boulard, Claire (2005): „The Spectator's Curtailed Legacy: The Periodical Press between England and France in the $18^{\text {th }}$ Century.“ In: Frédéric Ogée (ed.): ,, Better in France?". The Circulation of Ideas across the Channel in the Eighteenth Century. Lewisburg: Bucknell University Press, 144-161.

Boulard, Claire (2006): „Travestissement et stratégies narratives dans le Female Spectator d'Eliza Haywood.“ In: Guyonne Leduc (ed.): Travestissement féminin et liberté(s). Paris: L'Harmattan, 221-233.

Boulard, Claire/Ertler, Klaus-Dieter (eds.) (2020): Addison and Europe / Addison et l'Europe. Berlin [u.a.]: Peter Lang.

Brandstettner, Therese (2016): El Regañón General: Analyse einer Moralischen Wochenschrift aus Spanien. Universität Graz, Diplomarbeit.

Bravo, Francisco (1994): „Consideraciones sobre la mujer en dos periódicos gaditanos del siglo ilustrado: La Pensadora gaditana y La Academia de ociosos. “ In: Cinta Canterla (ed.): La mujer en los siglos XVIII y XIX. Actas del VII encuentro de la Ilustración al Romanticismo: Cádiz, América y Europa ante la modernidad; Cádiz 19, 20 y 21 de mayo de 1993. Cádiz: Universidad de Cádiz, 407-414.

Brenner, Peter J. (2015): „Reisen.“ In: Heinz Thoma (ed.): Handbuch europäische Aufklärung. Begriffe, Konzepte, Wirkung. Stuttgart [u.a.]: Metzler, 429-438. 
Brink, Margot (2008): „Geschlechterstreit und Dialektik der Aufklärung in Spanien und Frankreich. Die ambivalente Rolle von Vernunft und Natur in Egalitäts- und Komplementaritätstheorien des 18. Jahrhunderts.“ In: Friederike Hassauer (ed.): Heißer Streit und kalte Ordnung. Epochen der ,"Querelle des femmes “ zwischen Mittelalter und Gegenwart. Göttingen: Wallstein, 344-364.

Brockmeier, Jens/Harré, Rom (2005): „Die narrative Wende: Reichweite und Grenzen eines alternativen Paradigmas.“ In: Psychologie und Gesellschaftskritik 3-4/29, 3157.

Brokmann-Nooren, Christiane (1994): Weibliche Bildung im 18. Jahrhundert: ,gelehrtes Frauenzimmer" und ,gefällige Gattin “. Oldenburg: BIS Verlag.

Bührmann, Andrea D./Diezinger, Angelika/Metz-Göckel, Sigrid (2014): Arbeit - Sozialisation - Sexualität. Zentrale Felder der Frauen- und Geschlechterforschung. Wiesbaden: Springer.

Buijnsters, P.J. (1999a): „Le Misanthrope (1711-1712).“ In: Dictionnaire des Journaux 1600-1789, online: http://dictionnaire-journaux.gazettes18e.fr/journal/0958le-misanthrope, 25/09/19.

Buijnsters, P.J. (1999b): „Le Nouveau Spectateur français (1725-1726).“ In: Dictionnaire des Journaux 1600-1789, online: http://dictionnaire-journaux.gazettes18e.fr/journal/0993-le-nouveau-spectateur-francais, 14/09/19.

Burger, Lilith (2016): Des traitez de morale en guise de pieces divertissantes. Eine Untersuchung der von Justus van Effen aus dem Englischen übertragenen Moralischen Wochenschrift Le Mentor Moderne. Universität Graz, Diplomarbeit.

Butler, Judith (2012 [1991]): „Das Unbehagen der Geschlechter.“ In: Franziska Bergmann/Franziska Schößler/Bettina Schreck (eds.): Gender Studies. Bielefeld: transcript, 141-155.

Butler, Martin/Hausmann, Albrecht/Kirchhofer, Anton (2016): „Introduction: Participation and Precarious Alliances, Now and Then. “In: Martin Butler/Albrecht Hausmann/Anton Kirchhofer (eds.): Precarious Alliances. Cultures of Participation in Print and Other Media. Bielefeld: transcript, 7-14.

Calhoun, Bonnie (2012): „Shaping the Public Sphere: English Coffeehouses and French Salons and the Age of the Enlightenment.“ In: Colgate Academic Review 7/3, 75-99.

Canterla, Cinta (ed.) (1996): La Pensadora gaditana. Edición antológica. Cádiz: Universidad de Cádiz.

Cantos Casenave, Marieta (1995): „Orden y transgresión en la España ilustrada: La visión de El Argonauta.“ In: Alberto Romero Ferrer (ed.): De la Ilustración al romanticismo 1750-1850: VI encuentro „Juego, fiesta y transgresión “. Cádiz: Universidad de Cádiz, 463-477.

Capel Martínez, Rosa Ma (2010): „Prensa y Escritura Femenina en la España Ilustrada.“ In: El Argonauta español 7, online: http://journals.openedition.org/argonauta/431, 02/02/20.

Châtelet, Émilie du (1961 [1779]): Discours sur le bonheur. Édition critique et commentée par Robert Mauzi. Paris: Société d'édition Les Belles Lettres.

Chaudon, Louis Mayeul (ed.) (1804): Nouveau dictionnaire historique ou histoire abrégée de tous les hommes qui se sont fait un nom par des talens, des vertus, des forfaits, des erreurs, etc. 11. Sa-Th. Lyon: Bruyset. 
Coke, David (2017): Vauxhall Gardens 1661-1728. Brief History, online: http://www.vauxhallgardens.com/vauxhall_gardens_briefhistory_page.html, 04/04/18.

Conley, John (2002): The Suspicion of Virtue: Women Philosophers in Neoclassical France. Ithaca, NY: Cornell University Press.

Coquelet, Louis (1728): La méchante femme. Paris: Jacques Langlois.

Cotnam, Jacques/Hébert, Pierre (eds.) (2010): La gazette littéraire de Montréal, 17781779. Québec: Presses de 1'Université Laval.

Cowan, Brian (2004): „Mr. Spectator and the Coffeehouse Public Sphere.“ In: Eighteenth-Century Studies 3/37, 345-366.

Cyba, Eva (2010): „Patriarchat: Wandel und Aktualität.“ In: Ruth Becker/Beate Kortendiek (eds.): Handbuch Frauen- und Geschlechterforschung. Theorie, Methoden, Empirie. Wiesbaden: VS Verlag, 17-22.

Dale, Scott (ed.) (2005): La pensadora gaditana. Edición, introducción y notas de Scott Dale. Newark: Juan de la Cuesta.

Damasio, Antonio (2011): Selbst ist der Mensch: Körper, Geist und die Entstehung des menschlichen Bewusstseins. München: Siedler, epub.

DCMI (1995-2019): The Dublin Core Metadata Initiative, online: http://www.dublincore.org/, 29/07/19.

Defoe, Daniel (1702): Good advice to the Ladies shewing that as the world goes, and is like to go, the best way for them is to keep Unmarried. London: s.n.

Degner, Juliane/Meiser, Thorsten/Rothermund, Klaus (2009): „Kognitive und sozialkognitive Determinanten: Stereotype und Vorurteile.“ In: Andreas Beelmann/Kai J. Jonas (eds.): Diskriminierung und Toleranz. Psychologische Grundlagen und Anwendungsperspektiven. Wiesbaden: VS Verlag, 75-93.

Delacroix, Jacques-Vincent (1794): Le Spectateur françois pendant le gouvernement révolutionnaire. Paris: Buisson.

Devincenzo, Giovanna (2002): Marie de Gournay. Un cas littéraire. Fasano, Brindisi: Schena.

Diderot, Denis/D'Alembert, Jean-Baptiste le Rond (eds.) (2016): Encyclopédie, ou dictionnaire raisonné des sciences, des arts et des métiers, etc. University of Chicago: ARTFL Encyclopédie Project (Spring 2016 Edition), Robert Morrissey/ Glenn Roe (eds.), online: http://encyclopedie.uchicago.edu/, 25/09/19.

Döcker, Ulrike (1994): Die Ordnung der bürgerlichen Welt. Verhaltensideale und soziale Praktiken im 19. Jahrhundert. Frankfurt a. M. [u.a.]: Campus.

Domenech, Jacques (1997): „Vertu.“ In: Michel Delon (ed.): Dictionnaire européen des Lumières. Paris: Presses universitaires de France, 1085-1088.

Doms, Misia Sophia (ed.) (2020): Spectator-Type Periodicals in International Perspective. Enlightened Moral Journalism in Europe and North America. Frankfurt a. M.: Peter Lang.

Doms, Misia Sophia/Walcher, Bernhard (eds.) (2012): Periodische Erziehung des Menschengeschlechts. Moralische Wochenschriften im deutschsprachigen Raum. Bern: Peter Lang.

Eagleton, Terry (2005 [1984]): The Function of Criticism. London, New York: Verso.

Eaton, J. W. (1931): ,The French Influence in Denmark in the Seventeenth and Eighteenth Centuries." In: Germanic Review 4/6, 321-362. 
Einhoff, Eberhard (1980): Emanzipatorische Aspekte im Frauenbild von The Review, The Spectator und The Female Spectator. Frankfurt a. M.: Peter Lang.

Eisenhut, Ulrike (2011): „Literaturkritik in Moralischen Wochenschriften: The Tatler und seine französische Übersetzung Le philosophe nouvelliste." In: Klaus-Dieter Ertler (ed.): Die Spectators in der Romania - eine transkulturelle Gattung? Frankfurt a. M.: Peter Lang, 99-120.

Elias, Norbert (1997 [1939]): Über den Prozeß der Zivilisation. Soziogenetische und psychogenetische Untersuchungen. Band 1. Frankfurt a. M.: Suhrkamp.

Eliassen, Knut Ove (2010): „Remarks on the Historicity of the Media Concept.“ In: Ansgar Nünning/Vera Nünning/Birgit Neumann (eds.): Cultural Ways of Worldmaking. Media and Narratives. Berlin [u.a.]: de Gruyter, 119-135.

Engesser, Sven (2013): Die Qualität des Partizipativen Journalismus im Web. Bausteine für ein integratives theoretisches Konzept und eine explanative empirische Analyse. Wiesbaden: Springer.

Erll, Astrid (2005): „Literatur als Medium des kollektiven Gedächtnisses.“ In: Astrid Erll/Ansgar Nünning (eds.): Gedächtniskonzepte der Literaturwissenschaft. Theoretische Grundlegung und Anwendungsperspektiven. Berlin: de Gruyter, 249-276.

Ertler, Klaus-Dieter (2003a): Moralische Wochenschriften in Spanien. José Clavijo y Fajardo - „El Pensador “. Tübingen: Narr.

Ertler, Klaus-Dieter (2003b): „Die Perzeption des Eigenen und des Fremden als Konstanten eines idealtypischen aufklärerischen Diskursgefüges in Il Caffè. “In: Helmut C. Jacobs (ed.): Die Zeitschrift , Il caffe“ “. Vernunftprinzip und Stimmenvielfalt in der italienischen Aufklärung. Frankfurt a. M.: Peter Lang, 31-45.

Ertler, Klaus-Dieter (2004): Tugend und Vernunft in der Presse der spanischen Aufklärung: El Censor. Tübingen: Narr.

Ertler, Klaus-Dieter (2006): „Estrategias de argumentación en El Pensador de José Clavijo y Fajardo.“ In: Alberto Gil/Christian Schmitt (eds.): Rétorica en las lenguas iberorrománicas. Bonn: Romanistischer Verlag, 3-15.

Ertler, Klaus-Dieter (2008): „Entwürfe von Kommunikation in La Pensadora gaditana von Doña Beatriz Cienfuegos.“ In: Siegfried Jüttner (ed.): Die Konstituierung eines Kultur- und Kommunikationsraumes Europa im Wandel der Medienlandschaft des 18. Jahrhunderts. Frankfurt a. M.: Peter Lang, 1-11.

Ertler, Klaus-Dieter (2012a): „Moralische Wochenschriften.“ In: Europäische Geschichte Online (EGO), s.p.

Ertler, Klaus-Dieter (2012b): „Das Charakterbild in den Moralischen Wochenschriften - Justus Van Effens Le Misantrope." In: Angela Fabris/Willi Jung (eds.): Charakterbilder. Zur Poetik des literarischen Porträts. Göttingen: Vandenhoeck \& Ruprecht, 215-229.

Ertler, Klaus-Dieter (2014a): „Die Gattung der frankophonen „Spectators“ im Spiegel der zeitgenössischen Medienrevolution.“ In: Christof Schöch/Lars Schneider (eds.): PhiN. Philologie im Netz: Beihefte 7/2014 „Literaturwissenschaft im digitalen Medienwandel “, 18-35.

Ertler, Klaus-Dieter (2014b): „Du 'Spectateur' au 'Philosophe': Le prototype anglais dans les langues romanes." In: Bulletin d'Histoire Contemporaine de l'Espagne 49, 21-34.

Ertler, Klaus-Dieter (2014c): „Le système narratif des 'spectateurs' et leur réception en Espagne: quelques vecteurs discursifs dans le Pensador de José Clavijo y Fajar- 
do.“ In: El Argonauta español 11, online: http://argonauta.revues.org/2002, 08/01/15.

Ertler, Klaus-Dieter (ed.) (2011): Die Spectators in der Romania - eine transkulturelle Gattung? Frankfurt a. M.: Peter Lang.

Ertler, Klaus-Dieter (ed.) (2015): The Spectator Press: A Prominent Example of Cultural Heritage in the Construction of European Identity. EU-Antrag (Horizon 2020). Graz.

Ertler, Klaus-Dieter/Fuchs, Alexandra/Fischer, Michaela/Hobisch, Elisabeth/Scholger, Martina/Völkl, Yvonne (eds.) (2011-2020): Die ,,Spectators “ im internationalen Kontext. Universität Graz, Digitale Edition, online: https://gams.unigraz.at/mws, 25/09/19.

Ertler, Klaus-Dieter/Hobisch, Elisabeth (2014): Die „, Spectators “ in Spanien. Die kleinen Schriften der 1760er Jahre. Frankfurt a. M.: Peter Lang.

Ertler, Klaus-Dieter/Hobisch, Elisabeth/Humpl, Andrea Maria (2012): Die spanischen Spectators im Überblick. Frankfurt a. M.: Peter Lang.

Ertler, Klaus-Dieter/Hobisch, Elisabeth/Humpl, Andrea Maria (2014): Die Spectators in Spanien. Die kleinen Schriften der 1780er Jahre. Frankfurt a. M.: Peter Lang.

Ertler, Klaus-Dieter/Köhldorfer, Jessica (2010): Die „,Spectators“ in Spanien. „El duende especulativo sobre la vida civil" von Juan Antonio Mercadàl. Frankfurt a. M.: Peter Lang.

Ertler, Klaus-Dieter/Völkl, Yvonne/Hobisch, Elisabeth/Fuchs, Alexandra/Fernández, Hans (eds.) (2020): Storytelling in the Spectators/Storytelling dans les spectateurs. Frankfurt a. M.: Peter Lang.

Ette, Ottmar (2010): „ZwischenWelten der Literatur(wissenschaft): Auf dem Weg zu einer Poetik der Bewegung im Kontext der TransArea Studies." In: Doris Eibl/Gerhild Fuchs/Birgit Mertz-Baumgartner (eds.): Cultures à la dérive - cultures entre les rives. Grenzgänge zwischen Kulturen, Medien und Gattungen. Festschrift für Ursula Mathis-Moser zum 60. Geburtstag. Würzburg: Königshausen \& Neumann, 41-57.

Ette, Ottmar (2013): Viellogische Philologie. Die Literaturen der Welt und das Beispiel einer transarealen peruanischen Literatur. Berlin: Frey.

Evans, James E./Wall, John N. (1977): A Guide to Prose Fiction in the 'Tatler' and the 'Spectator'. New York: Garland.

Fabris, Angela (2011): „I fogli moralistici veneziani e le loro strategie: la Gazzetta Veneta di Gasparo Gozzi.“ In: Klaus-Dieter Ertler (ed.): Die Spectators in der Romania - eine transkulturelle Gattung? Frankfurt a. M.: Peter Lang, 165-186.

Fahrenwald, Claudia (2011): Erzählen im Kontext neuer Lernkulturen. Eine bildungstheoretische Analyse im Spannungsfeld von Wissen, Lernen und Subjekt. Wiesbaden: VS Verlag.

Faulstich, Werner (1998): Medien zwischen Herrschaft und Revolte. Die Medienkultur der frühen Neuzeit (1400-1700). Göttingen: Vandenhoeck \& Ruprecht.

Faulstich, Werner (2002): Die bürgerliche Mediengesellschaft (1700-1830). Göttingen: Vandenhoeck \& Ruprecht.

Feijoo, Benito Jerónimo (1726): Teatro crítico universal. Tomo 1. Texto tomado de la edición de Madrid 1778 (por D. Joaquín Ibarra, a costa de la Real Compañía de Impresores y Libreros), 325-398. 
Felbinger, Andrea (2004): Der Wandel des Bildungsbegriffes unter feministischer Perspektive. Auf den Spuren der Geschlechterbildung. München: Profil.

Fernández, Hans/Striedner, Pascal (eds.) (2019): Collecçam dos papeis anonymos. Berlin [u.a.]: Peter Lang.

Fischer, Michaela (2012): „Ami lecteur'. Le rôle du lecteur chez Marivaux.“ In: Klaus-Dieter Ertler/Alexis Lévrier/Michaela Fischer (eds.): Regards sur les 'spectateurs'. Periodical essay - Feuilles volantes - Moralische WochenschriftenFogli moralistici - Prensa moral. Frankfurt a. M.: Peter Lang, 97-117.

Fischer, Michaela (2014): Die Figur des Lesers im Kommunikationssystem der Spectateurs. Frankfurt a. M.: Peter Lang.

Fischer, Michaela (2016): Niederländische Moralische Wochenschriften. E-Mail. Graz.

Fischer-Pernkopf, Michaela (2019): „Lire La Spectatrice. Stratégies communicationelles dans le premier écrit spectatorial au féminin.“ In: Yvonne Völk1/Albert Göschl (eds.): Observations. Beobachtungen zu Literatur und Moral in der Romania und den Amerikas. Festschrift zum 65. Geburtstag von Klaus-Dieter Ertler. Wien: LIT, 49-61.

Fischer-Pernkopf, Michaela/Mussner, Veronika/Ertler, Klaus-Dieter (2018): Die «Spectators» in Frankreich. "Le Nouveau Spectateur» und «Le Monde comme il est» von Jean-François de Bastide. Frankfurt a. M.: Peter Lang.

Florack, Ruth (2001): Tiefsinnige Deutsche, frivole Franzosen. Nationale Stereotype in deutscher und französischer Literatur. Stuttgart [u.a.]: Metzler.

Florack, Ruth (2007): Bekannte Fremde. Zu Herkunft und Funktion nationaler Stereotype in der Literatur. Tübingen: Niemeyer.

Foucault, Michel (1977): Überwachen und Strafen. Die Geburt des Gefängnisses. Frankfurt a. M.: Suhrkamp.

Foucault, Michel (1983 [1976]): Der Wille zum Wissen. Sexualität und Wahrheit 1. Frankfurt a. M.: Suhrkamp.

Franchetti, Anna Lia (2002): „Introduction.“ In: Jean-Claude Arnould (ed.): Marie de Gournay. Euvres complètes. Tome 1. Paris: Champion, 9-97.

Frasponi, Cesare (1727) (ed.): „A chi legge.“ In: Il Filosofo alla Moda. Vol.1 $\backslash 000$, ediert in: Klaus-Dieter Ertler et al. (eds.) (2011-2020): Die „, Spectators “ im internationalen Kontext, Universität Graz, Digitale Edition, online: hdl.handle.net/11471/513.20.346, 20/02/19.

Frevert, Ute (1986): Frauen-Geschichte zwischen bürgerlicher Verbesserung und neuer Weiblichkeit. Frankfurt a. M.: Suhrkamp.

Friedrich, Hans-Edwin (ed.) (2006): Bürgerlichkeit im 18. Jahrhundert. Tübingen: Niemeyer.

Frye, Northrop (1971): „Littérature et mythe““ In: Poétiques 8, 489-503.

Fuchs, Alexandra (2011): Die Erzählstrategien in den italienischen Moralischen Wochenschriften „Il Filosofo alla Moda“, „L'Osservatore Veneto “ und „La Frusta letteraria di Aristarco Scannabue“. Universität Graz, Masterarbeit.

Fuchs, Alexandra (2016): Spuren der Moralischen Presse im Erzählwerk von Antonio Piazza. Frankfurt a. M.: Peter Lang.

Fuchs, Alexandra/Ertler, Klaus-Dieter/Holzer, Jürgen (eds.) (2019): Mikroerzählungen in den Spectators. Eine Anthologie. Hamburg: Dr. Kovač. 
Galleron, Iona (2018): „L'imaginaire de la perte dans La Bigarrure: regard authentiquement passéiste ou produit soigneusement marketé?" In: Klaus-Dieter Ertler/Samuel Baudry/Yvonne Völkl (eds.): Discourses on Economy in the Spectators / Discours sur l'économie dans les spectateurs. Frankfurt a. M.: Peter Lang, 215-228.

García Pandavenes, Elsa (ed.) (1972): „El Censor“ (1781-1787). Antología. Barcelona: Labor.

Gaudry-Hudson, Christine (1991): „L'absence au féminin ou le statut de la femme marivaudienne.“ In: Études françaises 2/27, 35-41.

Geier, Manfred (2012): Aufklärung. Das europäische Projekt. Reinbek bei Hamburg: Rowohlt.

Gelz, Andreas (2006): Tertulia. Literatur und Soziabilität im Spanien des 18. und 19. Jahrhunderts. Frankfurt a. M.: Vervuert.

Gelz, Andreas (2012): „El Pensador como ‘murmurador público’ - el escándalo de los semanarios morales y la esfera pública en la España del siglo XVIII.“ In: KlausDieter Ertler/Alexis Lévrier/Michaela Fischer (eds.): Regards sur les 'spectateurs'. Periodical essay - Feuilles volantes - Moralische Wochenschriften - Fogli moralistici - Prensa moral. Frankfurt a. M.: Peter Lang, 213-225.

Gildemeister, Regine (2010): „Doing Gender: Soziale Praktiken der Geschlechterunterscheidung. “ In: Ruth Becker/Beate Kortendiek (eds.): Handbuch Frauen- und Geschlechterforschung. Theorie, Methoden, Empirie. Wiesbaden: VS Verlag, 137145.

Gilot, Michel (1975): Les journaux de Marivaux. Itinéraire moral et accomplissement esthétique. Lille: Atelier Repr. des Thèses Univ.

Gipper, Andreas (2015): „Aufklärer.“ In: Heinz Thoma (ed.): Handbuch europäische Aufklärung. Begriffe, Konzepte, Wirkung. Stuttgart [u.a.]: Metzler, 56-66.

Goffman, Erving (1979): Gender Advertisements. New York: Harper \& Row.

Goodman, Nelson (1984 [1978]): Weisen der Welterzeugung. [engl. Orig: Ways of Worldmaking]. Frankfurt a. M.: Suhrkamp.

Göschl, Albert (2016): Die Logik des essayistischen Gedankens. Zur Analyse der italienischen Essayistik zwischen Fin de Siècle und Zweitem Weltkrieg vor dem Hintergrund der Gattungsgeschichte. Heidelberg: Universitätsverlag Winter.

Graeber, Wilhelm (1986): Moralistik und Zeitschriftenliteratur im frühen 18. Jahrhundert. Van Effens und Marivaux' Beitrag zur Entwicklung des frühaufklärerischen Menschenbildes. Frankfurt a. M.: Peter Lang.

Graeber, Wilhelm (2005): „Swift's First Voyages to Europe: His Impact on Eighteenth-Century France.“ In: Hermann Josef Real (ed.): The Reception of Jonathan Swift in Europe. London: Continuum, 5-16.

Grétry, André (1789): Aspasie. Oper.

Gronemann, Claudia (2008): „,Catones sin barbas y Licurgos con basquiñas“ - Fingierte (weibliche) Autorschaft und Normierung von Weiblichkeit: Spanische moralische Wochenschriften im europäischen Gattungszusammenhang.“ In: Siegfried Jüttner (ed.): Die Konstituierung eines Kultur- und Kommunikationsraumes Europa im Wandel der Medienlandschaft des 18. Jahrhunderts. Frankfurt a. M.: Peter Lang, 25-40.

Gronemann, Claudia (2011): „La Pensatriz Salmantina - ¿el enigma que sigue velándose? Eine Lektüre des Textfragments als Travestie.“ In: Klaus-Dieter Ertler (ed.): 
Die Spectators in der Romania - eine transkulturelle Gattung? Frankfurt a. M.: Peter Lang, 253-269.

Gronemann, Claudia (2013): Polyphone Aufklärung. Zur Textualität und Performativität der spanischen Geschlechterdebatten im 18. Jahrhundert. Frankfurt a. M.: Vervuert.

Gronemann, Claudia (2017): „Del lujo ostentoso a la ética del hombre sociable: ocio y sociabilidad en las Cartas marruecas de Cadalso." In: Robert Fajen/Andreas Gelz (eds.): Ocio y ociosidad en el siglo XVIII español e italiano. Ozio e oziosità nel Settecento italiano e spagnolo. Frankfurt a. M.: Klostermann, 207-226.

Guinard, Paul-Jacques (1973): La presse espagnole de 1737 à 1791. Formation et signification d'un genre. Paris: Centre de recherches hispaniques, Institut d'études hispaniques.

Gunia, Inke (2008): De la "poesía" a la "literatura". El cambio de los conceptos en la formación del campo literario español del siglo XVIII y principios del XIX. Frankfurt a. M.: Vervuert.

Habermas, Jürgen (1990 [1962]): Strukturwandel der Öffentlichkeit. Untersuchungen zu einer Kategorie der bürgerlichen Gesellschaft. Mit einem Vorwort zur Neuauflage 1990. Frankfurt a. M.: Suhrkamp.

Habermas, Jürgen (1995 [1989]): „Institutions of the Public Sphere.“ In: Oliver BoydBarrett (ed.): Approaches to Media. A Reader. London: Arnold, 235-244.

Haechler, Jean (2007): Les insoumises. 18 portraits de femmes exceptionnelles. Paris: Nouveau monde.

Hagengruber, Ruth (2017): History of Women Philosophers and Scientists, online: https://historyofwomenphilosophers.org, 25/09/19.

Hagengruber, Ruth/Hecht, Hartmut (eds.) (2019): Émilie du Châtelet und die deutsche Aufklärung. Wiesbaden: Springer.

Hahn, Hans Henning (ed.) (2002): Stereotyp, Identität und Geschichte. Die Funktion von Stereotypen in gesellschaftlichen Diskursen. Frankfurt a. M.: Peter Lang.

Hahn, Hans Henning/Hahn, Eva (2002): „Nationale Stereotypen. Plädoyer für eine historische Stereotypenforschung. “ In: Hans Henning Hahn (ed.): Stereotyp, Identität und Geschichte. Die Funktion von Stereotypen in gesellschaftlichen Diskursen. Frankfurt a. M.: Peter Lang, 17-56.

Halbwachs, Maurice (1925): Les cadres sociaux de la mémoire. Édition électronique: Les Classiques des Sciences Sociales, online: http://classiques.uqac.ca/classiques/Halbwachs_maurice/cadres_soc_memoire/cadres_sociaux_memoire.pdf, 17/11/09.

Hall, Ingrid (2003): „Our Challenge for the $21^{\text {st }}$ Century: A Cutting-Edge Relationship.“ In: Ursula Mathis-Moser (ed.): Österreich - Kanada. Beiträge zum Kulturund Wissenstransfer. 1990-2000. Innsbruck: Leopold-Franzens-Universität, 51-56.

Harth, Dietrich (1995): „Über die Bestimmung kultureller Vorurteile, Stereotypen und images in fiktionalen Texten." In: Wolfgang Kubin (ed.): Mein Bild in deinem Auge. Exotismus und Moderne; Deutschland - China im 20. Jahrhundert. Darmstadt: Wissenschaftliche Buchgesellschaft, 17-42.

Häseler, Jens (2011): „Le Spectateur français in der zeitgenössischen Presselandschaft.“ In: Klaus-Dieter Ertler (ed.): Die Spectators in der Romania - eine transkulturelle Gattung? Frankfurt a. M.: Peter Lang, 85-98. 
Hassauer, Friederike (1997): „Die Seele ist nicht Mann, nicht Weib. Stationen der Querelles des Femmes in Spanien und Lateinamerika vom 16. zum 18. Jahrhundert.“ In: Gisela Bock/Margarete Zimmermann (eds.): Jahrbuch für Frauenforschung. Band 2. Die europäische Querelle des Femmes. Geschlechterdebatten seit dem 15. Jahrhundert. Stuttgart: Metzler, 203-238.

Hassauer, Friederike (2008): „Einleitung: Heißer Streit und kalte Ordnung. Historizität und Systematizität der Querelle des femmes." In: Friederike Hassauer (ed.): Heißer Streit und kalte Ordnung. Epochen der „Querelle des femmes “ zwischen Mittelalter und Gegenwart. Göttingen: Wallstein, 11-46.

Haßler, Gerda (2011): „Die Moralischen Wochenschriften aus sprachwissenschaftlicher Sicht: narrative und begriffliche Darstellungsformen.“ In: Klaus-Dieter Ertler (ed.): Die Spectators in der Romania - eine transkulturelle Gattung? Frankfurt a. M.: Peter Lang, 15-37.

Haßler, Gerda (2012): „Das Thema Sprache in Wochenschriften und in Reflexionen der Herausgeber.“ In: Klaus-Dieter Ertler/Alexis Lévrier/Michaela Fischer (eds.): Regards sur les 'spectateurs'. Periodical essay - Feuilles volantes - Moralische Wochenschriften - Fogli moralistici - Prensa moral. Frankfurt a. M.: Peter Lang, 323-356.

Hausbichler, Beate (2018): „Attacken gegen Genderforschung in der politischen Arena. “ In: Der Standard (19/12/18), online: https://derstandard.at/2000094259480-1192182008549/Attacken-gegen-Genderforschung-inder-politischen-Arena, 19/12/18.

Hausen, Karin (1976): „Die Polarisierung der Geschlechtscharaktere. Eine Spiegelung der Dissoziation von Erwerbs- und Familienleben." In: Werner Conze (ed.): Sozialgeschichte der Familie in der Neuzeit Europas. Stuttgart: Klett-Cotta, 363-393.

Henry, Madeleine Mary (1995): Prisoner of History. Aspasia of Miletus and Her Biographical Tradition. New York: Oxford University Press.

Heringer, Hans Jürgen (2004): Interkulturelle Kommunikation. Grundlagen und Konzepte. Tübingen [u.a.]: Francke.

Heringer, Hans Jürgen (2012): Interkulturelle Kompetenz. Ein Arbeitsbuch mit interaktiver $C D$ und Lösungsvorschlägen. Tübingen [u.a.]: Francke.

Hertel-Mesenhöller, Heike (2001): Das Bild der Frau im spanischen Roman des 18. Jahrhunderts. Im Spannungsfeld von Lebenswirklichkeit und Fiktion. Frankfurt a. M.: Vervuert.

Hervás y Panduro, Lorenzo (1789): Historia de la vida del Hombre. Concepción, Nacimiento, Infancia y Niñez del Hombre. Tomo 1. Madrid: La Imprenta de Aznár.

Heße, Kristina (2006): „Die Stimme der Natur. Veränderungen in der Ordnung der Geschlechter im Kontext der spanischen Aufklärung.“ In: Judith Klinger/Susanne Thiemann (eds.): Geschlechtervariationen. Gender-Konzepte im Übergang zur Neuzeit. Potsdam: Universitätsverlag Potsdam, 299-322.

Heße, Kristina (2008): Männlichkeiten im Spanien der Aufklärung. Der Diskurs der moralischen Wochenschriften 'El Pensador', 'La Pensadora gaditana' und 'El Censor'. Berlin: Logos.

Heße, Kristina (2011): „,Der spanische Entwurf eines Ideals aufgeklärter Männlichkeit in den Moralischen Wochenschriften El Pensador und El Censor." In: Klaus-Dieter Ertler (ed.): Die Spectators in der Romania - eine transkulturelle Gattung? Frankfurt a. M.: Peter Lang, 235-251. 
Hettling, Manfred (2015): „Bürger/Bürgerlichkeit.“ In: Heinz Thoma (ed.): Handbuch europäische Aufklärung. Begriffe, Konzepte, Wirkung. Stuttgart [u.a.]: Metzler, 123-131.

Hillesheim, Ingrun (2013): Polyphonien der Vernunft - Zur Konstruktion und Dekonstruktion von Aufklärung in französischen und deutschen Briefromanen des 18. Jahrhunderts. Hamburg: Dr. Kovač.

Hirn, Lisz (2019): Geht's noch! Warum die konservative Wende für Frauen gefährlich ist. Wien: Molden.

Hirschfelder, Dagmar (2017): „Bildnis und Individuum im Zeitalter der Aufklärung.“ In: Daniel Hess/Dagmar Hirschfelder (eds.): Renaissance - Barock - Aufklärung. Kunst und Kultur vom 16. bis zum 18. Jahrhundert. Nürnberg, Heidelberg: Verlag des Germanischen Nationalmuseums, 362-373.

Hobisch, Elisabeth (2012): ,El Curioso Entretenido “ y „La Pensadora Gaditana “: un análisis contrastivo de las formas narrativas y los temas centrales. Universität Graz, Masterarbeit.

Hobisch, Elisabeth (2017): La forma epistolar en los espectadores españoles. Características y tipología de las cartas. Frankfurt a. M.: Peter Lang.

Hobisch, Elisabeth (2018): „Les stratégies publicitaires dans les lettres des 'spectateurs’ espagnols.“ In: Klaus-Dieter Ertler/Samuel Baudry/Yvonne Völkl (eds.): Discourses on Economy in the Spectators / Discours sur l'économie dans les spectateurs. Hamburg: Dr. Kovač, 199-214.

Hodab, Renate (2006): Marivaux' ,, Spectateur français “ vor dem Hintergrund der europäischen Wochenschriften des 18. Jahrhunderts. Universität Graz, Diplomarbeit.

Höfer, Anette/Keilhauer, Annette (1996): Femme. München: De Gruyter Oldenbourg.

Höfer, Anette/Reichardt, Rolf(1986): Honnête homme, Honnêteté, Honnêtes gens. Reprint 2014. München: De Gruyter Oldenbourg.

Honegger, Claudia (1992): Die Ordnung der Geschlechter: die Wissenschaften vom Menschen und das Weib, 1750-1850. Frankfurt a. M.: Campus.

Honegger, Claudia (2011): „Die kognitiven Prinzipien der neuen Wissenschaften vom Menschen und die Genese einer weiblichen Sonderanthropologie in Frankreich.“ In: Theresa Wobbe (ed.): Die gesellschaftliche Verortung des Geschlechts. Diskurse der Differenz in der deutschen und französischen Soziologie um 1900. Frankfurt a. M.: Campus, 93-113.

Horowitz, Maryanne Cline (1976): „Aristotle and Woman.“ In: Journal of the History of Biology 2/9, 183-213.

Hötzl, Christiane (2016): Le Babillard ou le Philosophe Nouvelliste - eine Vergleichsstudie: zur Bedeutung des Tatler für die französische Frühaufklärung. Universität Graz, Diplomarbeit.

Hühn, Peter (2012): „Event and Eventfulness.“ In: Peter Hühn et al. (eds.): The living handbook of narratology. Hamburg: Hamburg University, online: http://www.lhn.uni-hamburg.de/article/event-and-eventfulness, 21/06/17.

Imhof, Michael (2002): „Stereotypen und Diskursanalyse. Anregungen zu einem Forschungskonzept kulturwissenschaftlicher Stereotypenforschung.“ In: Hans Henning Hahn (ed.): Stereotyp, Identität und Geschichte. Die Funktion von Stereotypen in gesellschaftlichen Diskursen. Frankfurt a. M.: Peter Lang, 57-71. 
Iser, Wolfgang (1972): Der implizite Leser: Kommunikationsformen des Romans von Bunyan bis Beckett. München: Fink.

Italia, Iona (2008): „Fair-Sexing it. The Spectator on Women.“ In: Media History 3/14, 323-335.

Italian Women Writers, online: https://www.lib.uchicago.edu/efts/IWW, 25/09/19.

Jacobs, Eva et al. (eds.) (1979): Woman and Society in Eighteenth-Century France. Essays in Honour of John Stephenson Spink. London: Athlone Press.

Jacobs, Helmut C. (2008): „Unmittelbarkeit, Spontaneität und Zufall - Die Funktion der Phantasie in den Medien der spanischen Aufklärung." In: Siegfried Jüttner (ed.): Die Konstituierung eines Kultur- und Kommunikationsraumes Europa im Wandel der Medienlandschaft des 18. Jahrhunderts. Frankfurt a. M.: Peter Lang, 53-66.

Jannidis, Fotis (2010): „Methoden der computergestützten Textanalyse““ In: Vera Nünning (ed.): Methoden der literatur- und kulturwissenschaftlichen Textanalyse. Ansätze - Grundlagen - Modellanalysen. Stuttgart [u.a.]: Metzler, 109-132.

Jannidis, Fotis (2017): Digital Humanities. Eine Einführung. Stuttgart: Metzler.

Jenkins, Henry/Ito, Mizuko/Boyd, Danah (2016): Participatory Culture in a Networked Era. A Conversion on Youth, Learning, Commerce and Politics. Cambridge: Polity Press.

Jockers, Matthew Lee (2011): On Distant Reading and Macroanalysis, online: http://www.matthewjockers.net/2011/07/01/on-distant-reading-and-macroanalysis/, 12/09/18.

Jockers, Matthew Lee (2013): Macroanalysis. Digital Methods and Literary History. Urbana: University of Illinois Press.

Johnson-Laird, Philip N./Oatley, Keith (2016): „Emotions in Music, Literature, and Film.“ In: Lisa Feldman Barrett/Michael Lewis/Jeannette M. Haviland-Jones (eds.): Handbook of Emotions. New York: Guilford Press, 82-97.

Jüttner, Siegfried (1999): „Von der Schwierigkeit, Mythen stillzulegen: Spanische Literatur und Aufklärung in der deutschen Hispanistik. “ In: Iberoamericana (19772000) $2 / 23,5-38$.

Jüttner, Siegfried (2008): „Archive der Nationenbildung im Namen Europas. Die Presse im Spanien der Aufklärung als Medium nationaler Regeneration.“ In: Siegfried Jüttner (ed.): Die Konstituierung eines Kultur- und Kommunikationsraumes Europa im Wandel der Medienlandschaft des 18. Jahrhunderts. Frankfurt a. M.: Peter Lang, 67-109.

Jüttner, Siegfried (2011): „Heuristische Archive der Lebensführung oder die Sehnsucht nach moralischer Ordnung: Der Spectateur français von Marivaux." In: Klaus-Dieter Ertler (ed.): Die Spectators in der Romania - eine transkulturelle Gattung? Frankfurt a. M.: Peter Lang, 51-84.

Jüttner, Siegfried/Schlobach, Jochen (eds.) (1992): Europäische Aufklärung(en). Einheit und nationale Vielfalt. Hamburg: Meiner.

Kafker, Frank A. (1996): The Encyclopedists as a Group. A Collective Biography of the Authors of the Encyclopédie. Oxford: Voltaire Foundation.

Kant, Immanuel (1975): „Was ist Aufklärung?“ In: Ehrhard Bahr (ed.): Was ist Aufklärung? Thesen und Definitionen. Stuttgart: Reclam, 9-17.

Kawczyński, Max (1969 [1880]): Studien zur Literaturgeschichte des XVIII. Jahrhunderts. Moralische Zeitschriften. Hildesheim: Olms. 
Kay, Donald (1975): Short Fiction in 'The Spectator'. Alabama: University of Alabama Press.

Kenklies, Karsten (2015): „Erziehung/Bildung.“ In: Heinz Thoma (ed.): Handbuch europäische Aufklärung. Begriffe, Konzepte, Wirkung. Stuttgart [u.a.]: Metzler, 161171.

Kilian, Elena (2002): Bildung, Tugend, Nützlichkeit. Geschlechterentwürfe im spanischen Aufklärungsroman des späten 18. Jahrhunderts. Würzburg: Königshausen \& Neumann.

Kitts, Sally Ann (1995): The Debate on the Nature, Role an Influence of Woman in the Periodical Press in Eighteenth-Century Spain. Lewiston, N.Y.: E. Mellen Press.

Klapeer, Christine M. (2014): Perverse Bürgerinnen. Staatsbürgerschaft und lesbische Existenz. Bielefeld: transcript.

Klein, Christian/Martínez, Matías (2009): „Wirklichkeitserzählungen. Felder, Formen und Funktionen nicht-literarischen Erzählens.“ In: Christian Klein/Matías Martínez (eds.): Wirklichkeitserzählungen. Felder, Formen und Funktionen nicht-literarischen Erzählens. Stuttgart [u.a.]: Metzler, 1-13.

Knaller, Susanne (2007): Ein Wort aus der Fremde. Geschichte und Theorie des Begriffs Authentizität. Heidelberg: Winter.

Knaller, Susanne (ed.) (2013): Realität und Wirklichkeit in der Moderne. Literatur, Kunst, Fotografie und Film. Universität Graz, Digitale Edition, online: http://gams.uni-graz.at/context:reko, 27/01/20.

Köhler, Erich (2006): Vorlesungen zur Geschichte der französischen Literatur. Band 4. Frühaufklärung. 2. Aufl. ed. Dietmar Rieger. Online: urn:nbn:de:bsz:25-opus27940.

Köppe, Tilmann/Kindt, Tom (2014): Erzähltheorie. Eine Einführung. Stuttgart: Reclam.

Krefting, Ellen/Nøding, Aina/Ringvej, Mona Renate (eds.) (2015): Eighteenth-Century Periodicals as Agents of Change. Perspectives on Northern Enlightenment. Leiden: Brill.

Kreis, Karl-Wilhelm (1985): „Zum Diskurs über die Frau im 18. Jahrhundert: Antagonistische Weiblichkeitskonzepte im Zeitalter der spanischen Aufklärung." In: Iberoamericana (1977-2000) 2/3 (25/26)/9, 19-41.

Kühlmann, Wilhelm (2012): „Moralische Aufklärung im 18. Jahrhundert. Ziele, Medien, Aporien. “In: Misia Sophia Doms/Bernhard Walcher (eds.): Periodische Erziehung des Menschengeschlechts. Moralische Wochenschriften im deutschsprachigen Raum. Bern: Peter Lang, 15-46.

Kuhn, Thomas S. (1988): Die Struktur wissenschaftlicher Revolutionen. Zweite revidierte und um das Postskriptum von 1969 ergänzte Auflage. Frankfurt a. M.: Suhrkamp.

Kühne, Thomas (1996): „Männergeschichte als Geschlechtergeschichte.“ In: Thomas Kühne (ed.): Männergeschichte - Geschlechtergeschichte. Männlichkeit im Wandel der Moderne. Frankfurt a. M. [u.a.]: Campus, 7-30.

Labrador Herráiz, Carmen/Pablos Ramírez, Juan Carlos de (1989): La educación en los papeles periódicos de la Ilustración española. Madrid: Ministerio de Educación y Ciencia, Secretaría General Técnica, Centro de Publicaciones.

Labrosse, Claude/Rétat, Pierre (1985): L'instrument périodique. La fonction de la presse au XVIIIe siècle. Lyon: Presses Universitaires de Lyon. 
Laqueur, Thomas (2003 [1990]): Making Sex. Body and Gender from the Greeks to Freud. Cambridge [u.a.]: Harvard University Press.

Lauriol, Claude (1999): „La Spectatrice danoise (1748-1750).“ In: Dictionnaire des Journaux 1600-1789, online: http://dictionnaire-journaux.gazettes18e.fr/journal/1229-la-spectatrice-danoise, 12/04/18.

Le Collectif de Grenoble (1982): „Le journaliste masqué.“ In: Pierre Rétat (ed.): Le journalisme d'Ancien Régime. Question et propositions/ Table ronde CNRS, 12-13 juin 1981. Lyon: Presses Universitaires de Lyon, 285-313.

Le Guellec, Maud (2011): „De The Spectator a El filósofo a la moda: un caso de adaptación en la prensa del siglo XVIII.“ In: Dieciocho 1/34, 113-126.

Le Hunte, Bem/Golembiewski, Jan A. (2014): „Stories Have the Power to Save us. A Neurological Framework for the Imperative to Tell Stories."In: Arts and Social Sciences Journal 2/5, 73-76.

Lefèvre, Amandine (2013): „La Bigarrure, un journal à la page?“ In: Alexis Lévrier/ Adeline Wrona (eds.): Matière et esprit du journal. Du Mercure galant à Twitter. Paris: PUPS, 93-105.

Lejeune, Philippe (1996 [1975]): Le pacte autobiographique. Paris: Éditions du Seuil. Lerner, Gerda (1986): The Creation of Patriarchy. New York: Oxford University Press.

Lerner, Gerda (1993): The Creation of Feminist Consciousness. From the Middle Ages to Eighteen-Seventy. New York: Oxford University Press.

Lévrier, Alexis (2007): Les journaux de Marivaux et le monde des 'spectateurs'. Paris: PUPS.

Lévrier, Alexis (2013a): „Justus Van Effen, un 'passeur' entre les presses anglaise et française." In: Lise Andries et al. (eds.): Intellectual Journeys. The Translation of Ideas in Enlightenment England, France and Ireland. Oxford: Voltaire Foundation, 233-246.

Lévrier, Alexis (ed.) (2013b): La Spectatrice. Reims: Épure.

Lévrier, Alexis/Sgard, Jean (2012): „Van Effen et l'écriture autobiographique.“ In: Dix-huitième siècle 1/44, 503-517.

Lièvre, Éloïse (2003): „'Ceci n'est pas un journal'. Marivaux et les écrits périodiques.“ In: Franck Salaün (ed.): Marivaux subversif? Actes du colloque organisé par le Centre d'Étude du XVIIIe Siècle de Montpellier, 14-16 mars 2002, Université PaulValéry. Paris: Desjonquières, 184-196.

Link, Jürgen/Link-Heer, Ursula (1990): „Diskurs/Interdiskurs und Literaturanalyse.“ In: Zeitschrift für Linguistik und Literaturwissenschaft (LiLi) 77, 88-99.

Lippmann, Walter (1998 [1922]): Public Opinion. With a New Introduction by Michael Curtis. New Brunswick, NJ [u.a.]: Transaction.

Lodge, David (1992): The Art of Fiction. Illustrated from Classic and Modern Texts. New York: Viking.

Losada, José Manuel (2004): „Costumbrismo in Spanish Literature and Its European Analogues.“ 'In: Steven P. Sondrup/Virgil Nemoianu (eds.): Nonfictional Romantic Prose. Expanding Borders. Amsterdam [u.a.]: Benjamins, 333-346.

Losada, José Manuel (2013): El costumbrismo español y sus conexiones europeas. Online: https://www.researchgate.net/publication/255960285, 14/10/16.

Lüsebrink, Hans-Jürgen (2016 [2005]): Interkulturelle Kommunikation. Interaktion, Fremdwahrnehmung, Kulturtransfer. 4. Aufl. Stuttgart [u.a.]: Metzler. 
Lüsebrink, Hans-Jürgen/Haß, Annika (2013): „L'Almanach des Dames (1801-1840) als Medium weiblicher Geschmacksbildung und Forum >feministischer ten.“ In: Hans-Jürgen Lüsebrink/York-Gothart Mix (eds.): Französische Almanachkultur im deutschen Sprachraum (1700-1815). Gattungsstrukturen, komparatistische Aspekte, Diskursformen. Göttingen: Vandenhoeck \& Ruprecht, 279-307.

Maar, Elke (1995): Bildung durch Unterhaltung die Entdeckung des Infotainment in der Aufklärung. Hallenser und Wiener moralische Wochenschriften in der Blütezeit des Moraljournalismus, 1748-1782. Pfaffenweiler: Centaurus.

Mack, Peter (1994): „Addison's Essays as Models for Composition in School Anthologies and Textbooks of the Eighteenth and Nineteenth Centuries.“ In: Paradigm 13, s.p.

Mackie, Erin Skye (1997): Market à la Mode. Fashion, Commodity, and Gender in The Tatler and The Spectator. Baltimore, Md.: Johns Hopkins University Press.

Maihofer, Andrea (1994): „Geschlecht als Existenzweise. Einige kritische Anmerkungen zu aktuellen Versuchen zu einem neuen Verständnis von 'Geschlecht'." In: Katharina Pühl (ed.): Geschlechterverhältnisse und Politik. Frankfurt a. M.: Suhrkamp, 168-187.

Maihofer, Andrea (1995): Geschlecht als Existenzweise. Macht, Moral, Recht und Geschlechterdifferenz. Frankfurt a. M.: Helmer.

Mannheim, Karl (1928): „Das Problem der Generationen.“ In: Kölner Vierteljahreshefte für Soziologie 7, 157-185; 309-330.

Martens, Wolfgang (1968): Die Botschaft der Tugend. Die Aufklärung im Spiegel der deutschen moralischen Wochenschriften. Stuttgart: Metzler.

Martín Gaite, Carmen (1972): Usos amorosos del dieciocho en España. Madrid: Siglo XXI de España Editores.

Mason, Haydn (1979): „Women in Marivaux: Journalist to Dramatist.“ In: Eva Jacobs (ed.): Woman and Society in Eighteenth-Century France. Essays in Honour of John Stephenson Spink. London: Athlone Press, 42-54.

Matthews Grieco, Sara F. (1994): „Körper, äußere Erscheinung und Sexualität.“ In: Georges Duby/Michelle Perrot/Heide Wunder (eds.): Geschichte der Frauen: Frühe Neuzeit. Band 3. Frankfurt a. M.: Campus, 61-101.

Matzat, Wolfgang (2015): „Der Bürger und die Frau von Stand: La Nouvelle Héloïse und die Folgen. Überlegungen zum Verhältnis von Eros und Polis im französischund spanischsprachigen Roman.“ In: Stephan Leopold (ed.): Planet Rousseau. Zur heteronomen Genealogie der Moderne. Paderborn: Fink, 113-130.

Mauzi, Robert (1969): L'idee du bonheur dans la littérature et la pensée françaises au XVIIIe siècle. Paris: Colin.

Mayer, Pia Nanamé (2019): La Bigarure (1749-1753): Ein fiktiver Briefwechsel als Medium der Aufklärung in Frankreich. Universität Graz, Diplomarbeit.

Melton, James Van Horn (2001): The Rise of the Public in Enlightenment Europe. Cambridge [u.a.]: Cambridge University Press.

Mercier, Anne-Marie (2013): „Les échanges culturels entre Français et Anglais: vers une meilleure compréhension? L'exemple de la presse (L'Esprit des journaux) entre 1772 et 1785.“ In: Lise Andries et al. (eds.): Intellectual Journeys. The Translation of Ideas in Enlightenment England, France and Ireland. Oxford: Voltaire Foundation, 247-273. 
Mergel, Andreas (2005): „Benedict Andersons Imagined Communities: Zur Karriere eines erfolgreichen Konzepts. Nachwort zur Neuauflage 2005.“ In: Benedict Anderson: Die Erfindung der Nation. Frankfurt a. M. [u.a.]: Campus, 281-306.

Mergel, Ines et al. (2013): „Social Media - Hype oder Revolution?“ In: Ines Mergel et al. (eds.): Praxishandbuch Soziale Medien in der öffentlichen Verwaltung. Wiesbaden: Springer, 21-43.

Meuser, Michael (2010): „Geschlechtersoziologie.“ In: Georg Kneer/Markus Schroer (eds.): Handbuch Spezielle Soziologien. Wiesbaden: VS Verlag, 145-162.

Mimikama, online: www.mimikama.at, 25/09/19.

Mlitz, Andrea (2008): Dialogorientierter Journalismus. Leserbriefe in der deutschen Tagespresse. Konstanz: UVK.

Molière (1897 [1672]): Les femmes savantes. Herausgegeben v. Hermann Fritsche. Berlin: Weidmannsche Buchhandlung.

Moretti, Franco (2016): Distant Reading. Übersetzt v. Christine Pries. Konstanz: Konstanz University Press.

Moureau, François (1999): „Le Babillard 1 (1724-1735).“ In: Dictionnaire des Journaux 1600-1789, online: http://dictionnaire-journaux.gazettes18e.fr/journal/0137le-babillard-1, 25/09/19.

Müller, Mario (2018): 'Le Spectateur françois pendant le gouvernement révolutionnaire' (1794-95) von Jacques-Vincent Delacroix als Spiegel seiner Zeit: Analyse struktureller und thematischer Aspekte einer französischen Moralischen Wochenschrift. Universität Graz, Diplomarbeit.

Mussner, Veronika (2016): Die Moralischen Wochenschriften in Frankreich. Le Monde comme il est von Jean-François de Bastide als Spiegel seiner Zeit. Universität Graz, Masterarbeit.

N.N. (2012): „Birgitte Thott.“ In: The History of Nordic Women's Literature, Kvinfo: Kopenhagen, online: https://nordicwomensliterature.net/writers/thott-birgitte/, $05 / 01 / 20$.

N.N. (2018): „Frauen im Alten Testament.“ In: Bibelwissen.de, online: http://www.bibelwissenschaft.de/bibelkunde/themenkapitel-at/frauen-im-at/, 14/02/18.

Nablow, Ralph A. (1990): The Addisonian Tradition in France. Passion and Objectivity in Social Observation. Rutherford, NJ [u.a.]: Fairleigh Dickinson University Press.

Nagel, Joane (1998): „Masculinity and Nationalism: Gender and Sexuality in the Making of Nations.“ In: Ethnic and Racial Studies 2/21, 242-269.

Necker, Suzanne (2013 [1801]): „Sur un nouveau genre de Spectateur.“ In: Catriona Seth (ed.): La fabrique de l'intime. Mémoires et journaux de femmes du XVIIIe siècle. Paris: Robert Laffont, 253-259.

Neumann, Birgit (2009): Die Rhetorik der Nation in britischer Literatur und anderen Medien des 18. Jahrhunderts. Trier: WVT.

Neumann, Birgit/Nünning, Ansgar (2006): „Kulturelles Wissen und Intertextualität: Grundbegriffe und Forschungsansätze zur Kontextualisierung von Literatur.“ In: Marion Gymnich/Birgit Neumann/Ansgar Nünning (eds.): Kulturelles Wissen und Intertextualität. Theoriekonzeptionen und Fallstudien zur Kontextualisierung von Literatur. Trier: WVT, 3-28. 
Niedermeier, Michael (2015): „Landschaft/Garten.“ In: Heinz Thoma (ed.): Handbuch europäische Aufklärung. Begriffe, Konzepte, Wirkung. Stuttgart [u.a.]: Metzler, 323-334.

Niefanger, Susanne (1997): Schreibstrategien in Moralischen Wochenschriften. Formalstilistische, pragmatische und rhetorische Untersuchungen am Beispiel von Gottscheds 'Vernünfftigen Tadlerinnen'. Tübingen: Niemeyer.

Nora, Pierre (ed.) (1992): Les lieux de mémoire. Paris: Gallimard.

Norton, Brian Michael (2015): „The Spectator, Aesthetic Experience and the Modern Idea of Happiness.“ In: English Literature 1/2, 87-104.

Nünning, Ansgar (2010): „Making Events - Making Stories - Making Worlds: Ways of Worldmaking from a Narratological Point of View.“ In: Ansgar Nünning/Vera Nünning/Birgit Neumann (eds.): Cultural Ways of Worldmaking. Media and Narratives. Berlin [u.a.]: de Gruyter, 191-214.

Nünning, Ansgar (2013): „Wie Erzählungen Kulturen erzeugen: Prämissen, Konzepte und Perspektiven für eine kulturwissenschaftliche Narratologie.“ In: Alexandra Strohmaier (ed.): Kultur - Wissen - Narration. Perspektiven transdisziplinärer Erzählforschung für die Kulturwissenschaften. Bielefeld: transcript, 15-53.

Nünning, Ansgar (ed.) (2008): Metzler Lexikon. Literatur- und Kulturtheorie. Stuttgart, Weimar: Metzler.

Nünning, Ansgar/Nünning, Vera (2010): „Ways of Worldmaking as a Model for the Study of Culture: Theoretical Frameworks, Epistemological Underpinnings, New Horizons.“ In: Ansgar Nünning/Vera Nünning/Birgit Neumann (eds.): Cultural Ways of Worldmaking. Media and Narratives. Berlin [u.a.]: de Gruyter, 1-25.

Nünning, Ansgar/Nünning, Vera/Neumann, Birgit (eds.) (2010): Cultural Ways of Worldmaking. Media and Narratives. Berlin [u.a.]: de Gruyter.

Nünning, Ansgar/Rupp, Jan (2012): „'The Internet's New Storytellers': Merkmale, Typologien und Funktionen narrativer Genres im Internet aus gattungstheoretischer, narratologischer und medienkulturwissenschaftlicher Sicht." In: Ansgar Nünning et al. (eds.): Narrative Genres im Internet. Theoretische Bezugsrahmen, Mediengattungstypologie und Funktionen. Trier: WVT, 3-50.

Nünning, Vera (2013): „Narrativität als interdisziplinäre Schlüsselkategorie.“ In: Hans Georg Kräusslich/Wolfgang Schluchter (eds.): Marsilius-Kolleg 2011/12. Vierter Jahresbericht des Marsilius-Kollegs. 86-104, online: http://www.marsilius-kolleg.uni-heidelberg.de/publikationen/jahresbericht_2011_12.html, 20/11/19.

Nünning, Vera/Nünning, Ansgar (eds.) (2002): Erzähltheorie transgenerisch, intermedial, interdisziplinär. Trier: WVT.

Oberkampf, Walter (1934): Die zeitungskundliche Bedeutung der moralischen Wochenschriften: Ihr Wesen und ihre Bedeutung. Dresden: Risse Verlag.

Opitz-Belakhal, Claudia (2010): Geschlechtergeschichte. Frankfurt a. M. [u.a.]: Campus.

Österreichische Bundesregierung (2017): Regierungsprogramm 2017-2022 der Neuen Volkspartei und Freiheitlichen Partei Österreichs, online: https://www.dieneuevolkspartei.at/download/Regierungsprogramm.pdf, 08/05/19.

Pabst, Esther Suzanne (2007): Die Erfindung der weiblichen Tugend. Kulturelle Sinngebung und Selbstreflexion im französischen Briefroman des 18. Jahrhunderts. Göttingen: Wallstein. 
Pallares-Burke, Maria Lúcia (1994): „An Androgynous Observer in the EighteenthCentury Press. La Spectatrice, 1728-29.“ In: Women's History Review 3/3, 411435.

Pallares-Burke, Maria Lúcia (1996): „The Spectator Abroad: The Fascination of the Mask." In: History of European Ideas 1/22, 1-18.

Pallares-Burke, Maria Lúcia (2004): „A Spectator of the Spectators: Jacques-Vincent Delacroix.“ In: Hans-Jürgen Lüsebrink/Jeremy D. Popkin (eds.): Enlightenment, Revolution, and the Periodical Press. Oxford: Voltaire Foundation, 145-157.

Papenheim, Wilhelm (1930): Die Charakterschilderungen im 'Tatler', 'Spectator' und 'Guardian'. Ihr Verhältnis zu Theophrast, La Bruyère und den englischen character-writers des 17. Jahrhunderts. Leipzig: Tauchnitz.

Pečar, Andreas/Tricoire, Damien (2015): Falsche Freunde. War die Aufklärung wirklich die Geburtsstunde der Moderne? Frankfurt a. M. [u.a.]: Campus.

Pellegrin, Marie-Frédérique (2017): „Procédés d'inversion chez Poulain de la Barre: pour un concept d'efféminage.“ In: Philosophiques 2/44, 193-208.

Peterson, H. (1936): „Notes on the Influence of Addison's Spectator and Marivaux's Spectateur Français upon El Pensador." In: Hispanic Review 3/4, 256-263.

Peuchet, Jacques (1791): Encyclopédie méthodique. Jurisprudence. Tome 10. Paris: Panckoucke.

Pienaar, William James Bennie (1929): English Influences in Dutch Literature and Justus Van Effen as Intermediary. An Aspect of Eighteenth Century Achievement. Cambridge: Cambridge University Press.

Pila, Justine/Torremans, Paul (2016): European Intellectual Property Law. Oxford: Oxford University Press.

Pinson, Guillaume (2016): La culture médiatique francophone en Europe et en Amérique du Nord. De 1760 à la veille de la Seconde Guerre mondiale. Québec: Presses de l'Université Laval.

Piwnik, Marie-Hélène (1979): O Anónimo. Journal portugais du XVIIIe siècle (17521754). Paris: Fundação Calouste Gulbenkian.

Polanyi, Michael (1985): Implizites Wissen. Übersetzt v. Horst Brühmann. Frankfurt a. M.: Suhrkamp.

Poulain de La Barre, François (1676): De l'Égalité des deux sexes, discours physique et moral, où l'on voit l'importance de se défaire des Préjugez. Paris: Jean du Puis.

Pucci, Suzanne R. (2001): Sites of the Spectator. Emerging Literary and Cultural Practice in Eighteenth-Century France. Oxford: Voltaire Foundation.

Raquejo, Tonia (ed.) (1991): Joseph Addison. Los placeres de la imaginación y otros ensayos de The Spectator. Madrid: Visor.

Rau, Fritz (1980): Zur Verbreitung und Nachahmung des Tatler und Spectator. Heidelberg: Winter.

Reddy, Latha/Gershenson Smith, Rebecca (2002): Issuing Her Own: The Female Tatler. University of Michigan, online: http://umich.edu/ ece/student_projects/female_tatler/, 22/07/16.

Reichenberger, Andrea (2016): Émilie du Châtelets Institutions physiques. Über die Rolle von Prinzipien und Hypothesen in der Physik. Wiesbaden: Springer.

Reid, Martine (2016): „Introduction.“ In: Ángeles Sirvent Ramos/María Isabel Corbí Sáez/María Ángeles Llorca Tonda (eds.): Femmes auteurs du dix-huitième siècle. Nouvelles approches critiques. Paris: Honoré Champion, 9-22. 
Rétat, Pierre (ed.) (1982): Le journalisme d'Ancien Régime. Table ronde CNRS, 12-13 juin 1981. Lyon: Presses Universitaires de Lyon.

Rimbault, Caroline (1999): „La Spectatrice (1728-1729).“ In: Dictionnaire des Journaux 1600-1789, online: http://dictionnaire-journaux.gazettes $18 \mathrm{e} . f r / j o u r n a l / 1228$ la-spectatrice, $14 / 04 / 18$.

Romero, María (1994): „Una primera visión de cambio en la mujer del siglo XVIII, bajo los 'Pensamientos' de Beatriz Cienfuegos en La Pensadora gaditana.“ In: Cinta Canterla (ed.): La mujer en los siglos XVIII y XIX. Actas del VII encuentro de la Ilustración al Romanticismo: Cádiz, América y Europa ante la modernidad; Cádiz 19, 20 y 21 de mayo de 1993. Cádiz: Universidad de Cádiz, 609-618.

Rosenberg, Aubrey (1999): „Nicolas de Gueudeville (1652-1719).“ In: Dictionnaire des Journaux 1600-1789, online: http://dictionnaire-journalistes.gazettes 18e.fr/journaliste/375-nicolas-gueudeville, 01/09/19.

Rottensteiner, Josef (2017): El Catón Compostelano: un análisis del periódico moral. Universität Graz, Diplomarbeit.

Rousseau, Jean-Jacques (1754): Discours sur l'origine et les fondements de l'inégalité parmi les hommes. Édition électronique: Les Échos du Maquis (2011), online: https://philosophie.cegeptr.qc.ca/wp-content/documents/Discours-surlin\%C3\%A9galit\%C3\%A9-1754.pdf, 20/02/20.

Rousseau, Jean-Jacques (1762): Émile, ou de l'éducation. Édition électronique: Les Classiques des Sciences Sociales, online: http://classiques.uqac.ca/classiques/Rousseau_jj/emile/emile.html, 20/02/20.

Roussel, Pierre (1775): Système physique et moral de la femme, ou tableau philosophique de la Constitution, de l'Etat organique, du Tempérament, des Mours, \& des Fonctions propres au Sexe. Paris: Vincent.

Ruhe, Ernstpeter (1968): Untersuchungen zu den altfranzösischen Übersetzungen der Disticha Catonis. München: Max Hueber.

Salovey, Peter (2017): „Why we Need the Humanities More Than Ever.“ In: World Economic Forum, online: https://www.weforum.org/agenda/2017/03/the-key-toresponsible-and-responsive-leadership-the-humanities/, 18/06/17.

Sánchez Hita, Beatriz (2012): „Los espectadores y la prensa femenina. De la Pensadora Gaditana (1763-1764) al Amigo de las Damas (1813).“ In: Klaus-Dieter Ertler/Alexis Lévrier/Michaela Fischer (eds.): Regards sur les 'spectateurs'. Periodical essay - Feuilles volantes - Moralische Wochenschriften - Fogli moralistici - Prensa moral. Frankfurt a. M.: Peter Lang, 177-195.

Sánchez Hita, Beatriz (2019): „Espectadores y Duendes en los siglos XVIII y XIX. Entre la regeneración social y la reforma política. “In: Yvonne Völk1/Albert Göschl (eds.): Observations. Beobachtungen zu Literatur und Moral in der Romania und den Amerikas. Festschrift zum 65. Geburtstag von Klaus-Dieter Ertler. Wien: LIT, 103-115.

Sánchez-Blanco, Francisco (2016): El Censor. Un periódico contra el Antiguo Régimen. Sevilla: Ediciones Alfar.

Sanz Cabrerizo, Amelia (2016): „Initiatives pour la visibilité des femmes auteurs: e-archives.“ In: Ángeles Sirvent Ramos/María Isabel Corbí Sáez/María Ángeles Llorca Tonda (eds.): Femmes auteurs du dix-huitième siècle. Nouvelles approches critiques. Paris: Honoré Champion, 283-292. 
Sauder, Gerhard (1992): „Spielarten der Empfindsamkeit in England, Frankreich und Deutschland.“ In: Siegfried Jüttner/Jochen Schlobach (eds.): Europäische Aufklärung(en). Einheit und nationale Vielfalt. Hamburg: Meiner, 106-116.

Sauder, Gerhard (1997): „Sensibilité.“ In: Michel Delon (ed.): Dictionnaire européen des Lumières. Paris: Presses universitaires de France, 985-990.

Schaufler, Birgit (2002): „, Schöne Frauen - starke Männer“. Zur Konstruktion von Leib, Körper und Geschlecht. Opladen: Leske + Budrich.

Scheffel, Michael (2004): „Erzählen als anthropologische Universalie: Funktionen des Erzählens im Alltag und in der Literatur.“ In: Rüdiger Zymner (ed.): Anthropologie der Literatur. Poetogene Strukturen und ästhetisch-soziale Handlungsfelder. Paderborn: Mentis, 121-138.

Scheffel, Michael (2011): „Anthropologie des Erzählens.“ In: Matías Martínez (ed.): Handbuch Erzählliteratur. Theorie, Analyse, Geschichte. Stuttgart [u.a.]: Metzler, 74-79.

Schindlmeier, Lisa (2017): Die Entwicklung der Tugend und der Erziehung im 'Regañón General'. Universität Graz, Diplomarbeit.

Schneiders, Werner (1997): Das Zeitalter der Aufklärung. München: C.H. Beck.

Schöch, Christof (2013): „Big? Smart? Clean? Messy? Data in the Humanities.“ In: Journal of Digital Humanities 3/2, 2-13.

Schöch, Christof (2016): „Ein digitales Textformat für die Literaturwissenschaften. Die Richtlinien der Text Encoding Initiative und ihr Nutzen für Textedition und Textanalyse.“ In: Romanische Studien 4, 325-364.

Scholger et al. (2019): Distant Spectators: Distant Reading for Periodicals of the Enlightenment (DiSpecs). ÖAW-Projekt (go!digital Next Generation, 2019-2020). Graz. https://gams.uni-graz.at/dispecs, 10/01/20.

Scholger, Martina (2018): „'Spectators' in the International Context - A Digital Scholarly Edition.“ In: Klaus-Dieter Ertler/Samuel Baudry/Yvonne Völkl (eds.): Discourses on Economy in the Spectators / Discours sur l'économie dans les spectateurs. Hamburg: Dr. Kovač, 229-247.

Schorr, James L. (1982): The Life and Works of Justus van Effen. Wyoming: University of Wyoming.

Schorr, James L. (1986): Le Misanthrope. Oxford: Voltaire Foundation.

Schorr, James L. (2014): La Bagatelle (1718-1719). A Critical Edition of Justus Van Effen's Journal. Oxford: Voltaire Foundation.

Schümmer, Franz (1955): „Die Entwicklung des Geschmacksbegriffs in der Philosophie des 17. und 18. Jahrhunderts." In: Archiv für Begriffsgeschichte 1, 120-141.

Seethaler, Josef/Melischek, Gabriele (2008): „International vergleichende Mediengeschichte.“ In: Gabriele Melischek/Josef Seethaler/Jürgen Wilke (eds.): Medien \& Kommunikationsforschung im Vergleich. Grundlagen, Gegenstandsbereiche, Verfahrensweisen. Wiesbaden: VS Verlag, 43-73.

Semlak, Martina (2014): „Digitale Edition als Instrument für literaturwissenschaftliche Forschung.“ In: PhiN. Philologie im Netz: Beihefte 7/2014, 36-48.

Sermain, Jean-Paul (2011): „L'invention d'un périodique littéraire (Addison/Steele 1711-Marivaux 1721).“ In: Klaus-Dieter Ertler (ed.): Die Spectators in der Romania-eine transkulturelle Gattung? Frankfurt a. M.: Peter Lang, 39-50. 
Sgard, Jean (1999a): „Le Censeur (1714).“ In: Dictionnaire des Journaux 1600-1789, online: http://dictionnaire-journaux.gazettes18e.fr/journal/0202-le-censeur, 01/09/19.

Sgard, Jean (1999b): „Le Spectateur français 2 (1770?-1772).“ In: Dictionnaire des Journaux 1600-1789, online: http://dictionnaire-journaux.gazettes18e.fr/journal/1218-le-spectateur-francais-2, 04/04/18.

Sgard, Jean (ed.) (1999c): Dictionnaire des Journalistes. 1600-1789. Édition électronique revue, corrigée et augmentée. Paris: Universitas; Voltaire Foundation, online: http://dictionnaire-journalistes.gazettes18e.fr/, 19/02/20.

Sgard, Jean/Gilot, Michel (eds.) (1991): Dictionnaire des journaux. 1600-1789. Paris: Universitas; Voltaire Foundation.

Sgard, Jean/Gilot, Michel (eds.) (1999): Dictionnaire des Journaux. 1600-1789. Paris: Universitas; Voltaire Foundation, online: http://dictionnaire-journaux.gazettes $18 \mathrm{e}$.fr/, 10/02/20.

Shevelow, Kathryn (1989): Women and Print Culture: The Construction of Femininity in the Early Periodical. London: Routledge.

Sieuzac, Laurence (2009): „Éducation et vocation de la femme au Siècle des lumières.“ In: Paul Pasteur et al. (eds.): Genre \& Éducation. Former, se former, être formée au féminin. Mont-Saint-Aignan: Presses universitaires de Rouen et du Havre, 271287.

Smith, John Harrington (1952): „Thomas Baker and The Female Tatler.“ In: Modern Philology 3/49, 182-188.

Société Internationale pour l'Étude des Femmes de l'Ancien Régime, online: http://siefar.org, 25/09/19.

Stanzel, Franz K. (1998): Europäer. Ein imagologischer Essay. Heidelberg: Winter.

Stanzel, Franz K. (ed.) (1999): Europäischer Völkerspiegel. Imagologisch-ethnographische Studien zu den Völkertafeln des frühen 18. Jahrhunderts. Heidelberg: Winter.

Steinbrügge, Lieselotte (1987): Das moralische Geschlecht. Theorien und literarische Entwürfe über die Natur der Frau in der französischen Aufklärung. Weinheim: Beltz.

Steinbrügge, Lieselotte (1994): „'Qui peut définir les femmes?' L’idée de la 'nature féminine' au siècle des Lumières.“ In: Dix-huitième siècle 26, 333-348.

Stöber, Rudolf (2008): „Epochenvergleiche in der Medien- und Kommunikationsgeschichte.“ In: Gabriele Melischek/Josef Seethaler/Jürgen Wilke (eds.): Medien \& Kommunikationsforschung im Vergleich. Grundlagen, Gegenstandsbereiche, Verfahrensweisen. Wiesbaden: VS Verlag, 27-42.

Stolzenberg, Jürgen (2012): ,Joseph Addison.“ In: Monika Betzler/Maria-Doria Cojocaru/Julian Nida-Ruemelin (eds.): Ästhetik und Kunstphilosophie. In Einzeldarstellungen von der Antike bis zur Gegenwart. Stuttgart: Alfred Kröner Verlag, 1-5.

Striedner, Pascal (2018): Virtudes y vicios sociales en el periódico portugués ilustrado O Anonymo (1752-1754). Universität Graz, Masterarbeit.

Strömquist, Liv (2017): Der Ursprung der Welt. Berlin: avant-verlag.

Strömquist, Liv (2018): Der Ursprung der Liebe. Berlin: avant-verlag.

Stürzer, Volker (1984): Journalismus und Literatur im frühen 18. Jahrhundert. Die literarischen Beiträge in Tatler, Spectator und den anderen Blättern der Zeit. Frankfurt a. M.: Peter Lang. 
Sullerot, Evelyne (1966): Histoire de la Presse féminine en France des origines à 1848. Paris: Armand Colin.

Suppan, Arnold (1998): „Identitäten und Stereotypen in multiethnischen europäischen Regionen." In: Valeria Heuberger/Arnold Suppan/Elisabeth Vyslonzil (eds.): Das Bild vom Anderen. Identitäten, Mentalitäten, Mythen und Stereotypen in multiethnischen europäischen Regionen. Frankfurt a. M.: Peter Lang, 9-20.

Thiele, Martina (2015): Medien und Stereotype: Konturen eines Forschungsfeldes. Bielefeld: transcript.

Thomas, Antoine-Léonard (1772): Essai sur le caractère, les mours et l'esprit des femmes dans les différens siècles. Paris: Moutard.

Todorov, Tzvetan (1971 [1966]): „Typologie du roman policier.“ In: Tzvetan Todorov (ed.): Poétique de la prose. Paris: Éditions du Seuil, 55-65.

Tranow, Ulf (2016): „Norm, soziale.“ In: Johannes Kopp/Anja Steinbach (eds.): Grundbegriffe der Soziologie. Wiesbaden: Springer VS, 256-260.

Tschilschke, Christian von (2009): Identität der Aufklärung/Aufklärung der Identität. Literatur und Identitätsdiskurs im Spanien des 18. Jahrhunderts. Frankfurt a. M.: Vervuert.

Tschilschke, Christian von (2011): „Kulturspezifische Züge der Moralischen Wochenschriften in Spanien am Beispiel von El Censor.“ In: Klaus-Dieter Ertler (ed.): Die Spectators in der Romania - eine transkulturelle Gattung? Frankfurt a. M.: Peter Lang, 187-211.

Turner, Zelma Inez (1949): An Analysis of the Styles of Addison and Steele in the „Spectator" Papers. Atlanta University, Master Thesis.

Urzainqui Miqueleiz, Inmaculada (1995): „Autocreación y formas autobiográficas en la prensa crítica del siglo XVIII.“ In: Anales de Literatura Española 11, 193-226.

Urzainqui Miqueleiz, Inmaculada (2006): „Trabajadoras de la pluma: las periodistas en la época ilustrada.“ In: Josefina Méndez Vázquez (ed.): Maternidad, Familia y Trabajo: de la invisibilidad histórica de las mujeres a la igualdad contemporánea. Ávila: Fundación Sánchez Albornoz, 127-145.

Urzainqui Miqueleiz, Inmaculada (2012): „El ultimo ‘espectador’ español: El Regañón General de Ventura Ferrer." In: Klaus-Dieter Ertler/Alexis Lévrier/Michaela Fischer (eds.): Regards sur les 'spectateurs'. Periodical essay - Feuilles volantes Moralische Wochenschriften - Fogli moralistici - Prensa moral. Frankfurt a. M.: Peter Lang, 227-248.

Urzainqui Miqueleiz, Inmaculada (2016): „La prensa española como modeladora de la conducta femenina." In: Manuel-Reyes García Hurtado (ed.): El siglo XVIII en femenino. Las mujeres en el Siglo de las Luces. Madrid: Síntesis, 306-326.

Urzainqui, Inmaculada (2009): „Periodista-espectador en la España de las Luces. La conciencia de un género nuevo de escritura periodística. “In: El Argonauta español 6, online: http://journals.openedition.org/argonauta/516, 23/09/18.

Urzainqui, Inmaculada/La Ruiz de Peña, Juan Luis (1983): Periodismo e ilustración en Manuel Rubin de Celis. Oviedo: Centro de Estudios del Siglo XVIII.

Uzcanga Meinecke, Francisco (ed.) (2005): El Censor. Edición crítica. Barcelona: Crítica.

Valle-Inclán Alsina, Javier del (1990): „Noticias sobre el Caton compostelano y don Francisco del Valle-Inclán.“'In: Boletín de la ANABAD 2-3/40, 165-172.

van Delft, Louis (1982): Le moraliste classique. Genève: Librairie Droz. 
van Delft, Louis (2005a): Literatur und Anthropologie. Menschliche Natur und Charakterlehre. Münster: LIT.

van Delft, Louis (2005b): Les spectateurs de la vie. Généalogie du regard moraliste. Sainte-Foy (Québec): Presses de l’Université Laval.

van Dijk, Suzan [sic] (2016): „D’une écrivaine à l'autre: la base de données WomenWriters.“ In: Ángeles Sirvent Ramos/María Isabel Corbí Sáez/María Ángeles Llorca Tonda (eds.): Femmes auteurs du dix-huitième siècle. Nouvelles approches critiques. Paris: Honoré Champion, 225-243.

van Dijk, Suzanna (1988): Traces de femmes. Présence féminine dans le journalisme français du XVIIIe siècle. Amsterdam: APA, Holland University Press.

Vester, Heinz-Günter (2009): Kompendium der Soziologie I: Grundbegriffe. Wiesbaden: VS Verlag für Sozialwissenschaften.

Vollhardt, Friedrich (2006): „Die Bildung des Bürgers. Wissensvermittlung im Medium der Moralischen Wochenschrift.“ In: Hans-Edwin Friedrich (ed.): Bürgerlichkeit im 18. Jahrhundert. Tübingen: Niemeyer, 135-147.

Wagenknecht, Peter (2007): „Was ist Heteronormativität? Zu Geschichte und Gehalt des Begriffs." In: Jutta Hartmann et al. (eds.): Heteronormativität. Empirische Studien zu Geschlecht, Sexualität und Macht. Wiesbaden: VS Verlag, 17-34.

Wagner, Christoph (2001): „Porträt und Selbstbildnis.“ In: Richard van Dülmen (ed.): Entdeckung des Ich. Die Geschichte der Individualisierung vom Mittelalter bis zur Gegenwart. Köln/Wien: Böhlau, 79-106.

Warner, Michael (1991): „Introduction: Fear of a Queer Planet.“ In: Social Text 29, 317.

Warner, Michael (2002): „Publics and Counterpublics.“ In: Public Culture 1/14, 4990.

Weiß, Anna (2019): Rousseaus 'La Nouvelle Héloïse' vor dem Hintergrund der Moralischen Wochenschriften. Universität Graz, Masterarbeit.

Welzer, Harald (2002): Das kommunikative Gedächtnis. Eine Theorie der Erinnerung. München: C.H. Beck.

Werbemelder*in, online: https://werbemelder.in/, 25/09/19.

White, Hayden V. (1991 [1973]): Metahistory. Die historische Einbildungskraft im 19. Jahrhundert in Europa. Frankfurt a. M.: Fischer.

Wieland, Christoph Martin (1773): „Aspasia. Eine griechische Erzählung.“ In: Der deutsche Merkur, 120-135.

Wiesböck, Laura (2018): In besserer Gesellschaft. Der selbstgerechte Blick auf die Anderen. Wien: Kremayr \& Scheriau.

Wiesböck, Laura (2019): „Der weibliche Körper als Areal der Beschämung.“ In: Die Presse 30. Juli 2019, online: https://diepresse.com/home/meinung/gastkommen$\operatorname{tar} / 5667344 /$ Der-weibliche-Koerper-als-Areal-der-Beschaemung, 19/01/20.

Wilke, Jürgen (2008): Grundzüge der Medien- und Kommunikationsgeschichte. Köln, Wien [u.a.]: Böhlau.

Witthaus, Jan-Henrik (2012): Sozialisation der Kritik im Spanien des aufgeklärten Absolutismus. Frankfurt a. M: Klostermann.

Wolf, Werner (2013): „Was wäre, wenn wir nicht erzählen könnten? Dystopische Spekulationen und andere Reflexionen zur Relevanz des Narrativen und der Narratologie.“ In: Alexandra Strohmaier (ed.): Kultur - Wissen - Narration. Perspektiven 
transdisziplinärer Erzählforschung für die Kulturwissenschaften. Bielefeld: transcript, 55-73.

Women Writers, online: http://www.womenwriters.nl, 25/09/19.

Wunder, Heide (1988): „Von der frumkeit zur Frömmigkeit. Ein Beitrag zur Genese bürgerlicher Weiblichkeit (15.-17. Jahrhundert). “In: Ursula A. J. Becher/Jörn Rüsen (eds.): Weiblichkeit in geschichtlicher Perspektive. Fallstudien und Reflexionen zu Grundproblemen der historischen Frauenforschung. Frankfurt a. M.: Suhrkamp, 174-188.

Wuthenow, Ralph-Rainer (1974): Das erinnerte Ich. Europäische Autobiographie und Selbstdarstellung im 18. Jahrhundert. München: C.H. Beck.

Yanes, Elena C. (2013): „Valores discursivos del vocativo en la prensa espectadora española del siglo XVIII.“ In: Res Diachronicae 11, 16-36.

Zach, Wolfgang (2017 [1986]): Poetic Justice. Theorie und Geschichte einer literarischen Doktrin. Begriff - Idee - Komödienkonzeption. Berlin: de Gruyter.

Zaehle, Barbara (1998): „Knigges Umgang mit Menschen und seine Vorläufer (1933).“ In: Michael Schlott (ed.): Wirkungen und Wertungen. Adolph Freiherr Knigge im Urteil der Nachwelt (1796-1994); eine Dokumentensammlung. Göttingen: Wallstein, 166-173.

Zimmermann, Margarete (2005): Salon der Autorinnen. Französische „dames de lettres "vom Mittelalter bis zum 17. Jahrhundert. Berlin: Schmidt.

Zumthor, Paul (1983): Introduction à la poésie orale. Paris: Seuil.

Zymner, Rüdiger (ed.) (2010): Handbuch Gattungstheorie. Stuttgart: Metzler. 